\title{
Some properties of Wright-type generalized hypergeometric function via fractional calculus
}

\author{
Snehal B Rao ${ }^{1}$, Jyotindra C Prajapati ${ }^{2}$, Amitkumar D Patel ${ }^{3}$ and Ajay K Shukla ${ }^{4 *}$
}

\section{"Correspondence:}

ajayshukla2@rediffmail.com

${ }^{4}$ Department of Applied

Mathematics \& Humanities, S. V.

National Institute of Technology,

Surat, 395007, India

Full list of author information is

available at the end of the article

\begin{abstract}
This paper is devoted to the study of a Wright-type hypergeometric function (Virchenko, Kalla and Al-Zamel in Integral Transforms Spec. Funct. 12(1):89-100, 2001) by using a Riemann-Liouville type fractional integral, a differential operator and Lebesgue measurable real or complex-valued functions. The results obtained are useful in the theory of special functions where the Wright function occurs naturally. MSC: 33C20; 33E20; 26A33; 26A99

Keywords: fractional integral and differential operators; generalized hypergeometric function; Lebesgue measurable functions
\end{abstract}

\section{Introduction and preliminaries}

Special functions, particularly the hypergeometric function, play a very important role in solving numerous problems of mathematical physics, engineering and mathematical sciences [1-3].

The Gauss hypergeometric function is defined [4] as

$$
{ }_{2} F_{1}(a, b ; c ; z)=\sum_{k=0}^{\infty} \frac{(a)_{k}(b)_{k}}{(c)_{k} k !} z^{k} \quad(|z|<1, c \neq 0,-1,-2, \ldots) .
$$

The generalized hypergeometric function in a classical sense has been defined [5] as

$$
\begin{aligned}
{ }_{p} F_{q}\left[\begin{array}{c}
a_{1}, \ldots, a_{p} ; z \\
b_{1}, \ldots, b_{q}
\end{array}\right] & ={ }_{p} F_{q}\left[a_{1}, \ldots, a_{p} ; b_{1}, \ldots, b_{q} ; z\right] \\
& =\sum_{k=0}^{\infty} \frac{\left(a_{1}\right)_{k} \cdots\left(a_{p}\right)_{k}}{\left(b_{1}\right)_{k} \cdots\left(b_{q}\right)_{k}} \frac{z^{k}}{k !} \quad(p=q+1,|z|<1),
\end{aligned}
$$

where denominator parameters are neither zero nor negative integer.

Several generalizations of hypergeometric functions [6-13] etc. have been made and also motivated us to further investigate the topic. Virchenko et al. [7] defined the generalized hypergeometric function $\left({ }_{2} R_{1}^{\tau}(z)\right)$ in a different manner (throughout the paper, we call this

\section{Springer}

(C2014 Rao et al.; licensee Springer. This is an Open Access article distributed under the terms of the Creative Commons Attribution License (http://creativecommons.org/licenses/by/2.0), which permits unrestricted use, distribution, and reproduction in any medium, provided the original work is properly cited. 
function the Wright-type generalized hypergeometric function) as follows:

$$
{ }_{2} R_{1}^{\tau}(z)={ }_{2} R_{1}(a, b ; c ; \tau ; z)=\frac{\Gamma(c)}{\Gamma(b)} \sum_{k=0}^{\infty} \frac{(a){ }_{k} \Gamma(b+\tau k)}{\Gamma(c+\tau k) k !} z^{k} ; \quad \tau>0,|z|<1 .
$$

If $\tau=1$, then (3) reduces to a Gauss hypergeometric function ${ }_{2} F_{1}(a, b ; c ; z)$.

Rao et al. [14] obtained many properties for the function ${ }_{2} R_{1}(a, b ; c ; \tau ; z)$ as defined in (3) including the following result. If $a, b, c \in \mathbb{C} ; \operatorname{Re}(a)>0, \operatorname{Re}(b)>0, \operatorname{Re}(c)>0$, then

$$
\left(\frac{d}{d z}\right)^{m}\left[z^{c-1}{ }_{2} R_{1}\left(a, b ; c ; \tau ; \omega z^{\tau}\right)\right]=z^{c-m-1} \frac{\Gamma(c)}{\Gamma(c-m)}{ }_{2} R_{1}\left(a, b ; c-m ; \tau ; \omega z^{\tau}\right) .
$$

Prajapati et al. [12], Prajapati and Shukla [15] and Srivastava et al. [16] used the fractional calculus approach in the study of an integral operator and also generalized the MittagLeffler function.

The subject of fractional calculus [17-20] deals with the investigations of integrals and derivatives of any arbitrary real or complex order, which unify and extend the notions of integer-order derivative and $n$-fold integral. It has gained importance and popularity during the last four decades or so, mainly due to its vast potential of demonstrated applications in various seemingly diversified fields of science and engineering, such as fluid flow, rheology, diffusion, relaxation, oscillation, anomalous diffusion, reaction-diffusion, turbulence, diffusive transport, electric networks, polymer physics, chemical physics, electrochemistry of corrosion, relaxation processes in complex systems, propagation of seismic waves, dynamical processes in self-similar and porous structures. Recently some interesting results on fractional boundary value problems and fractional partial differential equations were also discussed by Nyamoradi et al. [21] and Baleanu et al. [22, 23].

In continuation of the study on the significance of fractional calculus, we define the integral operator as follows:

$$
\left(R_{\alpha+;, \tau, c}^{\omega ; a, b} f\right)(x)=R_{\alpha+; \tau, c}^{\omega ; a, b} f(x)=\int_{\alpha}^{x}(x-t)^{c-1} R\left(a, b ; c ; \tau ; \omega(x-t)^{\tau}\right) f(t) d t \quad(x>\alpha),
$$

where, $a, b, c, \omega \in \mathbb{C} ; \operatorname{Re}(a)>0, \operatorname{Re}(b)>0, \operatorname{Re}(c)>0 ; \tau>0$.

Substituting $\tau=1$, (5) reduces to the operator

$$
F_{\alpha+; c}^{\omega ; a, b} f(x)=\int_{\alpha}^{x}(x-t)^{c-1}{ }_{2} F_{1}\left(a, b ; c ; \omega(x-t)^{\tau}\right) f(t) d t, \quad x>\alpha .
$$

First, we give preliminaries, notations and definitions.

$L(\alpha, \beta)$ is the space of Lebesgue measurable real or complex-valued functions such that

$$
L(\alpha, \beta)=\left\{f:\|f\|_{1} \equiv \int_{\alpha}^{\beta}|f(t)| d t<\infty\right\}
$$

The Gauss multiplication formula [4] is given as follows. If $m$ is a positive integer and $z \in \mathbb{C}$, then

$$
\prod_{k=1}^{m} \Gamma\left(z+\frac{k-1}{m}\right)=(2 \pi)^{(m-1) / 2} m^{\frac{1}{2}-m z} \Gamma(m z) .
$$


The representation of a generalized factorial function in terms of the Pochhammer symbol [24] is given for

$$
\lambda \neq 0,-1,-2, \ldots ; \quad(\lambda)_{n}=\frac{\Gamma(\lambda+n)}{\Gamma(\lambda)}= \begin{cases}1, & \text { if } n=0, \\ \lambda(\lambda+1) \cdots(\lambda+n-1), & \text { if } n=1,2,3, \ldots\end{cases}
$$

for

$$
\lambda=0,-1,-2, \ldots ; \quad(\lambda)_{n}= \begin{cases}\frac{(-1)^{n}(-\lambda) !}{(-\lambda-n) !}, & \text { if } 0 \leq n \leq(-\lambda) \\ 0, & \text { if } n>(-\lambda) .\end{cases}
$$

Integration and differentiation of fractional order are traditionally defined by the leftsided Riemann-Liouville fractional integral operator $I_{\alpha+}^{\mu}$ and the right-sided RiemannLiouville fractional integral operator $I_{\beta-}^{\mu} f$ and the corresponding Riemann-Liouville fractional derivative operators $D_{\alpha+}^{\mu} f$ and $D_{\alpha-}^{\mu} f[3,17]$, which are given as follows.

If $f(x) \in L(\alpha, \beta), \mu \in \mathbb{C}, \operatorname{Re}(\mu)>0$, then

$$
{ }_{\alpha} D_{x}^{-\mu} f(x)={ }_{\alpha} I_{x}^{\mu} f(x)=I_{\alpha+}^{\mu} f(x)=\left(I_{\alpha+}^{\mu} f\right)(x)=\frac{1}{\Gamma(\mu)} \int_{\alpha}^{x} \frac{f(t)}{(x-t)^{1-\mu}} d t \quad(x>\alpha)
$$

is called the Riemann-Liouville left-sided fractional integral of order $\mu$.

Analogously,

$$
{ }_{x} D_{\beta}^{-\mu} f(x)={ }_{x} I_{\beta}^{\mu} f(x)=I_{\beta-}^{\mu} f(x)=\left(I_{\beta-}^{\mu} f\right)(x)=\frac{1}{\Gamma(\mu)} \int_{x}^{\beta} \frac{f(t)}{(t-x)^{1-\mu}} d t \quad(x<\beta)
$$

is called the Riemann-Liouville right-sided fractional integral of order $\mu$.

For $\mu \in \mathbb{C}, \operatorname{Re}(\mu)>0 ; n=[\operatorname{Re}(\mu)]+1$, the left-sided and right-sided Riemann-Liouville fractional derivatives are defined as

$$
\begin{aligned}
& \left(D_{\alpha+}^{\mu} f\right)(x)=\left(\frac{d}{d x}\right)^{n}\left(I_{\alpha+}^{n-\mu} f\right)(x), \\
& \left(D_{\alpha-}^{\mu} f\right)(x)=\left(-\frac{d}{d x}\right)^{n}\left(I_{\alpha-}^{n-\mu} f\right)(x),
\end{aligned}
$$

respectively. Here $[x]$ denotes the maximal integer not exceeding real $x$.

A generalization of the Riemann-Liouville fractional derivative operator $D_{\alpha+}^{\mu}(13)$ has been made by introducing the fractional derivative operator $D_{\alpha+}^{\mu, v}$ of order $0<\mu<1$ and type $0 \leq v \leq 1$ with respect to $x$ as follows [18]:

$$
\left(D_{\alpha+}^{\mu, v} f\right)(x)=\left(I_{\alpha+}^{\nu(1-\mu)} \frac{d}{d x}\left(I_{\alpha+}^{(1-v)(1-\mu)} f\right)\right)(x)
$$

This equation (15) easily reduces to the classical Riemann-Liouville fractional derivative operator $D_{\alpha+}^{\mu}$ when $v=0$. Moreover, in its special case when $v=1$, (15) reduces to the Caputo fractional derivative operator.

The left- and right-sided Caputo fractional derivatives of order $\alpha \in \mathbb{C}(\operatorname{Re}(\alpha) \geq 0)$, denoted by $\left({ }^{C} D_{a+}^{\alpha} y\right)(x)$ and $\left({ }^{C} D_{b-}^{\alpha} y\right)(x)$ respectively, are defined on $[a, b]$ via the Riemann- 
Liouville fractional derivatives as

$$
\left({ }^{C} D_{a+}^{\alpha} y\right)(x):=\left(D_{a+}^{\alpha}\left[y(t)-\sum_{k=0}^{n-1} \frac{y^{k}(a)}{k !}(t-a)^{k}\right]\right)(x)
$$

and

$$
\left({ }^{C} D_{b-}^{\alpha} y\right)(x):=\left(D_{b-}^{\alpha}\left[y(t)-\sum_{k=0}^{n-1} \frac{y^{k}(b)}{k !}(b-t)^{k}\right]\right)(x),
$$

where, $n=[\operatorname{Re}(\alpha)]+1$ for $\alpha \notin \mathbb{N}_{0} ; n=\alpha$ for $\alpha \in \mathbb{N}_{0}$.

The following facts are prepared for our study.

Theorem 1.1 (Mathai and Haubold [20]) If $\mu, \beta \in \mathbb{C}, \operatorname{Re}(\mu)>0, \operatorname{Re}(\beta)>0$, then

$$
I_{\alpha+}^{\mu}(x-\alpha)^{\beta-1}=\frac{\Gamma(\beta)}{\Gamma(\mu+\beta)}(x-\alpha)^{\mu+\beta-1} .
$$

Theorem 1.2 (Srivastava and Manocha [24]) If a function $f(z)$, analytic in the disc $|z|<R$, has the power series expansion $f(z)=\sum_{n=0}^{\infty} a_{n} z^{n}(|z|<R)$, then

$$
{ }_{0} D_{z}^{-\mu}\left\{z^{\lambda-1} f(z)\right\}=\frac{\Gamma(\lambda)}{\Gamma(\lambda+\mu)} z^{\lambda+\mu-1} \sum_{n=0}^{\infty} \frac{a_{n}(\lambda)_{n}}{(\lambda+\mu)_{n}} z^{n}
$$

provided that $\operatorname{Re}(\lambda)>0, \operatorname{Re}(\mu)>0$ and $|z|<R$.

Lemma 1.1 The following result (Srivastava and Tomovski [16]) holds true for the fractional derivative operator $D_{\alpha+}^{\mu, v} f$ defined by (13) as

$$
\left(D_{\alpha+}^{\mu, v}\left[(t-\alpha)^{\lambda-1}\right]\right)(x)=\frac{\Gamma(\lambda)}{\Gamma(\lambda-\mu)}(x-\alpha)^{\lambda-\mu-1},
$$

where $x>\alpha ; 0<\mu<1 ; 0 \leq v \leq 1 ; \operatorname{Re}(\lambda)>0$.

\section{Main results}

Theorem 2.1 If $\alpha \in \mathbb{R}_{+}=[0, \infty), a, b, c, \mu, \omega \in \mathbb{C}, \operatorname{Re}(a)>0, \operatorname{Re}(b)>0, \operatorname{Re}(c)>0, \operatorname{Re}(\mu)>0$, $\tau>0$, then for $x>\alpha, \omega \in \mathbb{C}$ and $\tau,\left|\omega(x-\alpha)^{\tau}\right|<1$,

$$
\begin{aligned}
I_{\alpha+}^{\mu} & {\left[(x-\alpha)^{c-1}{ }_{2} R_{1}\left(a, b ; c ; \tau ; \omega(x-\alpha)^{\tau}\right)\right] } \\
& =\frac{(x-\alpha)^{\mu+c-1} \Gamma(c)}{\Gamma(c+\mu)}{ }_{2} R_{1}\left(a, b ; c+\mu ; \tau ; \omega(x-\alpha)^{\tau}\right), \\
D_{\alpha+}^{\mu} & {\left[(x-\alpha)^{c-1}{ }_{2} R_{1}\left(a, b ; c ; \tau ; \omega(x-\alpha)^{\tau}\right)\right] } \\
& =\Gamma(c)\left\{\frac{(x-\alpha)^{c-\mu-1}}{\Gamma(c-\mu)}{ }_{2} R_{1}\left(a, b ; c-\mu ; \tau ; \omega(x-\alpha)^{\tau}\right)\right\} .
\end{aligned}
$$

If $0<\mu<1,0 \leq v \leq 1$, then

$$
\begin{aligned}
& \left(D_{\alpha+}^{\mu, v}\left[(t-\alpha)_{2}^{c-1} R_{1}\left(a, b ; c ; \tau ; \omega(t-\alpha)^{\tau}\right)\right]\right)(x) \\
& \quad=(x-\alpha)^{c-\mu-1} \frac{\Gamma(c)}{\Gamma(c-\mu)}{ }_{2} R_{1}\left(a, b ; c-\mu ; \tau ; \omega(x-\alpha)^{\tau}\right) .
\end{aligned}
$$


Proof

$$
\begin{aligned}
I_{\alpha+}^{\mu} & {\left[(x-\alpha)^{c-1}{ }_{2} R_{1}\left(a, b ; c ; \tau ; \omega(x-\alpha)^{\tau}\right)\right] } \\
& =\frac{1}{\Gamma(\mu)} \int_{\alpha}^{x} \frac{(t-\alpha)^{c-1}{ }_{2} R_{1}\left(a, b ; c ; \tau ; \omega(t-\alpha)^{\tau}\right)}{(x-t)^{1-\mu}} d t \\
& =\frac{1}{\Gamma(\mu)} \frac{\Gamma(c)}{\Gamma(b)} \sum_{k=0}^{\infty} \frac{(a)_{k} \Gamma(b+\tau k)}{\Gamma(c+\tau k) k !} \omega^{k}\left(\int_{\alpha}^{x} \frac{(t-\alpha)^{c-1}}{(x-t)^{1-\mu}}(t-\alpha)^{\tau k} d t\right) \\
& =\frac{1}{\Gamma(\mu)} \frac{\Gamma(c)}{\Gamma(b)} \sum_{k=0}^{\infty} \frac{(a)_{k} \Gamma(b+\tau k)}{\Gamma(c+\tau k) k !} \omega^{k}\left(\int_{\alpha}^{x} \frac{(t-\alpha)^{c-1+\tau k}}{(x-t)^{1-\mu}} d t\right) \\
& =\frac{\Gamma(c)}{\Gamma(b)} \sum_{k=0}^{\infty} \frac{(a)_{k} \Gamma(b+\tau k)}{\Gamma(c+\tau k) k !} \omega^{k} I_{\alpha+}^{\mu}\left[(x-\alpha)^{c-1+\tau k}\right] .
\end{aligned}
$$

The use of (18) gives

$$
\begin{aligned}
I_{\alpha+}^{\mu} & {\left[(x-\alpha)^{c-1}{ }_{2} R_{1}\left(a, b ; c ; \tau ; \omega(x-\alpha)^{\tau}\right)\right] } \\
& =\frac{\Gamma(c)}{\Gamma(b)} \sum_{k=0}^{\infty} \frac{(a)_{k} \Gamma(b+\tau k)}{\Gamma(c+\tau k) k !} \omega^{k} \frac{\Gamma(c+\tau k)}{\Gamma(c+\mu+\tau k)}(x-\alpha)^{\mu+c+\tau k-1} \\
& =\frac{(x-\alpha)^{\mu+c-1} \Gamma(c)}{\Gamma(c+\mu)}\left(\frac{\Gamma(c+\mu)}{\Gamma(b)} \sum_{k=0}^{\infty} \frac{(a)_{k} \Gamma(b+\tau k)}{\Gamma(c+\mu+\tau k)} \frac{\left(\omega(x-\alpha)^{\tau}\right)^{k}}{k !}\right) \\
& =\frac{(x-\alpha)^{\mu+c-1} \Gamma(c)}{\Gamma(c+\mu)}{ }_{2} R_{1}\left(a, b ; c+\mu ; \tau ; \omega(x-\alpha)^{\tau}\right) .
\end{aligned}
$$

This completes the proof of (21).

From (22) and (13), we get

$$
\begin{aligned}
D_{\alpha+}^{\mu} & {\left[(x-\alpha)^{c-1}{ }_{2} R_{1}\left(a, b ; c ; \tau ; \omega(x-\alpha)^{\tau}\right)\right] } \\
& =\left(\frac{d}{d x}\right)^{n}\left\{I_{\alpha+}^{n-\mu}\left[(x-\alpha)^{c-1}{ }_{2} R_{1}\left(a, b ; c ; \tau ; \omega(x-\alpha)^{\tau}\right)\right]\right\}
\end{aligned}
$$

and, using (21), this takes the following form:

$$
\begin{aligned}
D_{\alpha+}^{\mu} & {\left[(x-\alpha)^{c-1}{ }_{2} R_{1}\left(a, b ; c ; \tau ; \omega(x-\alpha)^{\tau}\right)\right] } \\
& =\left(\frac{d}{d x}\right)^{n}\left[\frac{(x-\alpha)^{n-\mu+c-1} \Gamma(c)}{\Gamma(c+n-\mu)}{ }_{2} R_{1}\left(a, b ; c+n-\mu ; \tau ; \omega(x-\alpha)^{\tau}\right)\right] .
\end{aligned}
$$

Applying (4) gives

$$
=\Gamma(c)\left\{\frac{(x-\alpha)^{c-\mu-1}}{\Gamma(c-\mu)}{ }_{2} R_{1}\left(a, b ; c-\mu ; \tau ; \omega(x-\alpha)^{\tau}\right)\right\} .
$$

This is the proof of (22). 
We have

$$
\begin{aligned}
& \left(D_{\alpha+}^{\mu, v}\left[(t-\alpha)^{c-1}{ }_{2} R_{1}\left(a, b ; c ; \tau ; \omega(t-\alpha)^{\tau}\right)\right]\right)(x) \\
& \quad=\left(D_{\alpha+}^{\mu, v}\left[\frac{\Gamma(c)}{\Gamma(b)} \sum_{k=0}^{\infty} \frac{(a)_{k} \Gamma(b+\tau k)}{\Gamma(c+\tau k)} \frac{\omega^{k}}{k !}(t-\alpha)^{c+\tau k-1}\right]\right)(x) \\
& \quad=\frac{\Gamma(c)}{\Gamma(b)} \sum_{k=0}^{\infty} \frac{(a)_{k} \Gamma(b+\tau k)}{\Gamma(c+\tau k)} \frac{\omega^{k}}{k !} \cdot\left(D_{\alpha+}^{\mu, v}\left[(t-\alpha)^{c+\tau k-1}\right]\right)(x) ;
\end{aligned}
$$

and using the identity (20) yields

$$
\begin{aligned}
& \left(D_{\alpha+}^{\mu, \nu}\left[(t-\alpha)^{c-1}{ }_{2} R_{1}\left(a, b ; c ; \tau ; \omega(t-\alpha)^{\tau}\right)\right]\right)(x) \\
& =\frac{\Gamma(c)}{\Gamma(b)} \sum_{k=0}^{\infty} \frac{(a)_{k} \Gamma(b+\tau k)}{\Gamma(c+\tau k)} \frac{\omega^{k}}{k !} \cdot\left(\frac{\Gamma(c+\tau k)}{\Gamma(c+\tau k-\mu)}(x-\alpha)^{c+\tau k-\mu-1}\right) \\
& =(x-\alpha)^{c-\mu-1} \frac{\Gamma(c)}{\Gamma(c-\mu)}\left\{\frac{\Gamma(c-\mu)}{\Gamma(b)} \sum_{k=0}^{\infty} \frac{(a)_{k} \Gamma(b+\tau k)}{\Gamma(c-\mu+\tau k)} \frac{\left(\omega(x-\alpha)^{\tau}\right)^{k}}{k !}\right\} \\
& =(x-\alpha)^{c-\mu-1} \frac{\Gamma(c)}{\Gamma(c-\mu)}{ }_{2} R_{1}\left(a, b ; c-\mu ; \tau ; \omega(x-\alpha)^{\tau}\right) .
\end{aligned}
$$

This completes proof of the required assertion (23).

Corollary If $\mu, a, \lambda \in \mathbb{C}$ and $\tau>0$, then

$$
{ }_{0} I_{x}^{\mu}\left[\lambda_{2} R_{1}\left(a, 1 ; 1 ; \tau, \lambda x^{\tau}\right)\right]=\frac{\lambda x^{\mu}}{\Gamma(\mu+1)}{ }_{2} R_{1}\left(a, 1 ; \mu+1 ; \tau ; \lambda x^{\tau}\right)
$$

$\lambda \in \mathbb{C}$ is such that for chosen $x$ and $\tau,\left|\lambda x^{\tau}\right|<1$.

Proof The result can be obtained directly by multiplying (21) by $\lambda$ and taking $\alpha=0, b=1$, $c=1, \omega=\lambda$. Remarks:

(i) This corollary can also be obtained from result (11) as given in [25] and also from result (13) as given in [26], by putting $k=1$.

(ii) We obtain the results (21) and (22) in a different manner. These can also be obtained from results (11) and (14) as given in [25] and also from (13) and (15) as given in [26].

(iii) Riemann-Liouville fractional integrals of order $\mu$ for ${ }_{2} R_{1}(a, b ; c ; \tau ; z)$ and ${ }_{2} F_{1}(a, b ; c ; z)$ can be easily got by using Theorem 1.2 as

$$
I_{0+}^{\mu}\left\{{ }_{2} R_{1}(a, b ; c ; \tau ; z)\right\}=\left\{\sum_{n=0}^{\infty} \frac{(a)_{n}(b)_{\tau n}}{(c)_{\tau n}} \frac{1}{\Gamma(1+\mu+n)} z^{n+\mu}\right\}
$$

and

$$
I_{0+}^{\mu}\left\{{ }_{2} F_{1}(a, b ; c ; z)\right\}=\left\{\sum_{n=0}^{\infty} \frac{(a)_{n}(b)_{n}}{(c)_{n}} \frac{1}{\Gamma(1+\mu+n)} z^{n+\mu}\right\} .
$$


Theorem 2.2 For $\tau=m \in \mathbb{N}$, the generalized hypergeometric function ${ }_{2} R_{1}(a, b ; c ; \tau ; z)$ takes the form

$$
{ }_{2} R_{1}(a, b ; c ; m ; z)=\frac{(2 \pi)^{(m-1) / 2}}{m^{c-\frac{1}{2}}} \frac{\Gamma(c)}{\Gamma(b)} \prod_{k=0}^{m-1}\left(\frac{1}{\Gamma\left(\frac{c+k}{m}\right)} \sum_{n=0}^{\infty} \frac{(a)_{n} \Gamma(b+m n)}{\left(\frac{c+k}{m}\right)_{n}} \frac{z^{n}}{n ! m^{m n}}\right)
$$

$a, b, c \in \mathbb{C}, \operatorname{Re}(a)>0, \operatorname{Re}(b)>0, \operatorname{Re}(c)>0,|z|<1$.

Proof Putting $z=n+\frac{c}{m}$ in (8), we obtain

$$
\frac{1}{\Gamma(m z)}=\frac{1}{\Gamma(c+m n)}=\frac{(2 \pi)^{(m-1) / 2}}{m^{m z-\frac{1}{2}}} \frac{1}{\prod_{k=1}^{m} \Gamma\left(z+\frac{k-1}{m}\right)}=\frac{(2 \pi)^{(m-1) / 2}}{m^{c-\frac{1}{2}}} \frac{1}{m^{m n} \prod_{k=0}^{m-1} \Gamma\left(n+\frac{c+k}{m}\right)} .
$$

Thus,

$$
\frac{1}{\Gamma(c+m n)}=\frac{(2 \pi)^{(m-1) / 2}}{m^{c-\frac{1}{2}}} \frac{1}{m^{m n} \prod_{k=0}^{m-1} \Gamma\left(n+\frac{c+k}{m}\right)} .
$$

From (26) and (3) afterwards, $\tau=m \in \mathbb{N}$, we get

$$
\begin{aligned}
{ }_{2} R_{1}(a, b ; c ; m ; z) & =\frac{\Gamma(c)}{\Gamma(b)} \sum_{n=0}^{\infty}\left(\frac{(a)_{n} \Gamma(b+m n)}{m^{m n} \prod_{k=0}^{m-1} \Gamma\left(n+\frac{c+k}{m}\right)} \frac{(2 \pi)^{(m-1) / 2}}{m^{c-\frac{1}{2}}} \frac{z^{n}}{n !}\right) \\
& =\frac{(2 \pi)^{(m-1) / 2}}{m^{c-\frac{1}{2}}} \frac{\Gamma(c)}{\Gamma(b)} \prod_{k=0}^{m-1}\left(\frac{1}{\Gamma\left(\frac{c+k}{m}\right)} \sum_{n=0}^{\infty} \frac{(a)_{n} \Gamma(b+m n)}{\left(\frac{c+k}{m}\right)_{n}} \frac{z^{n}}{n ! m^{m n}}\right) .
\end{aligned}
$$

This is a proof of the result.

On putting $m=1$, this reduces to ${ }_{2} F_{1}(a, b ; c ; z)$.

\section{Some properties of the operator $\left(R_{\alpha+; \tau, c}^{\omega ; a, b} f\right)(x)$}

Theorem 3.1 If $a, b, c, \mu, \omega \in \mathbb{C} ; \operatorname{Re}(a)>0, \operatorname{Re}(b)>0, \operatorname{Re}(c)>0 ; \operatorname{Re}(\mu)>0 ; \tau>0$, then

$$
\begin{aligned}
& \left(R_{\alpha+;, c}^{\omega ; a, b}(t-\alpha)^{\mu-1}\right)(x) \\
& \quad=(x-\alpha)^{c+\mu-1} \Gamma(\mu) \frac{\Gamma(c)}{\Gamma(c+\mu)}{ }_{2} R_{1}\left(a, b ; c+\mu ; \tau ; \omega(x-\alpha)^{\tau}\right) .
\end{aligned}
$$

Proof From (5)

$$
\left(R_{\alpha+\tau, c}^{\omega ; a, b} f\right)(x)=\int_{\alpha}^{x}(x-t)^{c-1} R\left(a, b ; c ; \tau ; \omega(x-t)^{\tau}\right) f(t) d t \quad(x>\alpha) .
$$

Therefore,

$$
\begin{aligned}
& \left(R_{\alpha+\tau, c}^{\omega ; a, b}(t-\alpha)^{\mu-1}\right)(x) \\
& \quad=\int_{\alpha}^{x}(x-t)^{c-1} R\left(a, b ; c ; \tau ; \omega(x-t)^{\tau}\right)(t-\alpha)^{\mu-1} d t
\end{aligned}
$$




$$
\begin{aligned}
& =\frac{\Gamma(c)}{\Gamma(b)} \sum_{k=0}^{\infty} \frac{(a)_{k} \Gamma(b+\tau k)}{\Gamma(c+\tau k) k !} \omega^{k}\left(\int_{\alpha}^{x}(t-\alpha)^{\mu-1}(x-t)^{\tau k+c-1} d t\right) \\
& =\frac{\Gamma(c)}{\Gamma(b)} \sum_{k=0}^{\infty} \frac{(a)_{k} \Gamma(b+\tau k)}{\Gamma(c+\tau k) k !} \omega^{k}\left((x-\alpha)^{c+\tau k+\mu-1} \beta(c+\tau k, \mu)\right) \\
& =(x-\alpha)^{c+\mu-1} \Gamma(\mu) \frac{\Gamma(c)}{\Gamma(c+\mu)}\left\{\frac{\Gamma(c+\mu)}{\Gamma(b)} \sum_{k=0}^{\infty} \frac{(a)_{k} \Gamma(b+\tau k)}{\Gamma(c+\mu+\tau k)} \frac{\left(\omega(x-\alpha)^{\tau}\right)^{k}}{k !}\right\} \\
& =(x-\alpha)^{c+\mu-1} \Gamma(\mu) \frac{\Gamma(c)}{\Gamma(c+\mu)}{ }_{2} R_{1}\left(a, b ; c+\mu ; \tau ; \omega(x-\alpha)^{\tau}\right),
\end{aligned}
$$

this leads to the proof.

Theorem 3.2 If $a, b, c, \omega \in \mathbb{C} ; \operatorname{Re}(a)>0, \operatorname{Re}(b)>0, \operatorname{Re}(c)>0 ; \tau>0$ and $\beta>\alpha$, then the operator $R_{\alpha+; \tau, c}^{\omega ; a, b}$ is bounded on $L(\alpha, \beta)$ and

$$
\left\|R_{\alpha+;, c}^{\omega ; a, b} f\right\|_{1} \leq B\|f\|_{1}
$$

where

$$
B=(\beta-\alpha)^{\operatorname{Re}(c)} \sum_{k=0}^{\infty} \frac{\left|(a)_{k}\right|\left|(b)_{\tau k}\right|}{\left|(c)_{\tau k}\right|[\tau k+\operatorname{Re}(c)]} \frac{\left|\omega(\beta-\alpha)^{\tau}\right|^{k}}{k !} .
$$

Proof From (5) and (7), afterwards interchanging the order of integration by applying the Dirichlet formula [19], we obtain

$$
\begin{aligned}
\left\|R_{\alpha+\tau, c}^{\omega ; a, b} f\right\|_{1} & =\int_{\alpha}^{\beta}\left|\int_{\alpha}^{x}(x-t)^{c-1}{ }_{2} R_{1}\left(a, b ; c ; \tau ; \omega(x-t)^{\tau}\right) f(t) d t\right| d x \\
& \leq \int_{\alpha}^{\beta}\left[\int_{t}^{\beta}(x-t)^{\operatorname{Re}(c)-1}\left|{ }_{2} R_{1}\left(a, b ; c ; \tau ; \omega(x-t)^{\tau}\right)\right| d x\right]|f(t)| d t
\end{aligned}
$$

and substituting $(x-t)=u$, we have

$$
=\int_{\alpha}^{\beta}\left[\int_{0}^{\beta-t}(u)^{\operatorname{Re}(c)-1}{ }_{2} R_{1}\left(a, b ; c ; \tau ; \omega(u)^{\tau}\right) \mid d u\right]|f(t)| d t .
$$

Using (3) and further simplification gives

$$
\left\|R_{\alpha+;, c}^{\omega ; a, b} f\right\|_{1} \leq \int_{\alpha}^{\beta}\left[\sum_{k=0}^{\infty} \frac{\left|(a)_{k}\right|\left|(b)_{\tau k}\right|}{\left|(c)_{\tau k}\right|} \frac{\left|\omega^{k}\right|}{k !}\left(\frac{u^{\tau k+\operatorname{Re}(c)}}{\tau k+\operatorname{Re}(c)}\right)_{0}^{\beta-\alpha}\right]|f(t)| d t
$$

This equation can also be written as

$$
\begin{aligned}
& =\left\{(\beta-\alpha)^{\operatorname{Re}(c)}\left[\sum_{k=0}^{\infty} \frac{\left|(a)_{k}\right|\left|(b)_{\tau k}\right|}{\left|(c)_{\tau k}\right|[\tau k+\operatorname{Re}(c)]} \frac{\left|\omega(\beta-\alpha)^{\tau}\right|^{k}}{k !}\right]\right\} \cdot \int_{\alpha}^{\beta}|f(t)| d t \\
& =B\|f\|_{1} ;
\end{aligned}
$$


where

$$
B=(\beta-\alpha)^{\operatorname{Re}(c)}\left[\sum_{k=0}^{\infty} \frac{\left|(a)_{k}\right|\left|(b)_{\tau k}\right|}{\left|(c)_{\tau k}\right|[\tau k+\operatorname{Re}(c)]} \frac{\left|\omega(\beta-\alpha)^{\tau}\right|^{k}}{k !}\right] .
$$

This completes the proof of (28).

Theorem 3.3 If $a, b, c, \mu, \omega \in \mathbb{C} ; \operatorname{Re}(a)>0, \operatorname{Re}(b)>0, \operatorname{Re}(c)>0 ; \tau>0$ and $\beta>\alpha$, then

$$
\left(I_{\alpha+}^{\mu}\left[R_{\alpha+; \tau, c}^{\omega ; a, b} f\right]\right)(x)=\frac{\Gamma(c)}{\Gamma(c+\mu)}\left(R_{\alpha+; \tau, c+\mu}^{\omega ; a, b} f\right)(x)=\left(R_{\alpha+;, c}^{\omega ; a, b}\left[I_{\alpha+}^{\mu} f\right]\right)(x)
$$

holds for any summable function $f \in L(\alpha, \beta)$.

Proof From (11) and (5), we have

$$
\begin{aligned}
& \left(I_{\alpha+}^{\mu}\left[R_{\alpha+; \tau, c}^{\omega ; a, b} f\right]\right)(x) \\
& \quad=\frac{1}{\Gamma(\mu)} \int_{\alpha}^{x} \frac{\left[\left(R_{\alpha+\tau, c}^{\omega ; a, b} f\right)(t)\right]}{(x-t)^{1-\mu}} d t \\
& \quad=\frac{1}{\Gamma(\mu)} \int_{\alpha}^{x}(x-t)^{\mu-1}\left(\int_{\alpha}^{t}(t-u)^{c-1}{ }_{2} R_{1}\left(a, b ; c ; \tau ; \omega(t-u)^{\tau}\right) f(u) d u\right) d t .
\end{aligned}
$$

Interchanging the order of integration and using the Dirichlet formula [19], we get

$$
\begin{aligned}
& \left(I_{\alpha+}^{\mu}\left[R_{\alpha+; \tau, c}^{\omega ; a, b} f\right]\right)(x) \\
& \quad=\int_{\alpha}^{x}\left[\frac{1}{\Gamma(\mu)} \int_{u}^{x}(x-t)^{\mu-1}(t-u)^{c-1}{ }_{2} R_{1}\left(a, b ; c ; \tau ; \omega(t-u)^{\tau}\right) d t\right] f(u) d u .
\end{aligned}
$$

Substituting $(t-u)=\lambda$, we get

$$
\begin{aligned}
\left(I_{\alpha+}^{\mu}\right. & {\left.\left[R_{\alpha ; j, \tau, c}^{\omega ; a, b} f\right]\right)(x) } \\
\quad= & \int_{\alpha}^{x}\left[\frac{1}{\Gamma(\mu)} \int_{0}^{x-u}(x-u-\lambda)^{\mu-1}(\lambda)^{c-1}{ }_{2} R_{1}\left(a, b ; c ; \tau ; \omega(\lambda)^{\tau}\right) d \lambda\right] f(u) d u \\
\quad= & \int_{\alpha}^{x}\left[\frac{1}{\Gamma(\mu)} \int_{0}^{x-u} \frac{(\lambda)^{c-1}{ }_{2} R_{1}\left(a, b ; c ; \tau ; \omega(\lambda)^{\tau}\right)}{((x-u)-\lambda)^{1-\mu}} d \lambda\right] f(u) d u .
\end{aligned}
$$

Making the use of (11) and applying (21) yield

$$
\left(I_{\alpha+}^{\mu}\left[R_{\alpha+; \tau, c}^{\omega ; a, b} f\right]\right)(x)=\frac{\Gamma(c)}{\Gamma(c+\mu)} \int_{\alpha}^{x}\left[(x-u)^{\mu+c-1}{ }_{2} R_{1}\left(a, b ; c+\mu ; \tau ; \omega(x-u)^{\tau}\right)\right] f(u) d u .
$$

Thus, $\left(I_{\alpha+}^{\mu}\left[R_{\alpha+; \tau, c}^{\omega ; a, b} f\right]\right)(x)=\frac{\Gamma(c)}{\Gamma(c+\mu)} R_{\alpha+; \tau, c+\mu}^{\omega ; a, b} f(x)$.

This is the proof of the first part of (30). 
For proving the second part of the theorem, we start from the right-hand side of (30) and, using (5), we get

$$
\begin{aligned}
\left(R_{\alpha+\tau, c}^{\omega ; a, b}\left[I_{\alpha+}^{\mu} f\right]\right)(x) & =\int_{\alpha}^{x}(x-t)^{c-1} R_{1}\left(a, b ; c ; \tau ; \omega(x-t)^{\tau}\right)\left(I_{\alpha+}^{\mu} f\right)(t) d t \\
& =\int_{\alpha}^{x}(x-t)^{c-1}{ }_{2} R_{1}\left(a, b ; c ; \tau ; \omega(x-t)^{\tau}\right)\left(\frac{1}{\Gamma(\mu)} \int_{\alpha}^{t} \frac{f(u)}{(t-u)^{1-\mu}} d u\right) d t .
\end{aligned}
$$

Using the Dirichlet formula [19] and interchanging the order of integration, we have

$$
\begin{aligned}
& \left(R_{\alpha+; \tau, c}^{\omega ; a, b}\left[I_{\alpha+}^{\mu} f\right]\right)(x) \\
& \quad=\int_{u=\alpha}^{x} \frac{1}{\Gamma(\mu)}\left[\int_{t=u}^{x}(x-t)^{c-1}(t-u)^{\mu-1}{ }_{2} R_{1}\left(a, b ; c ; \tau ; \omega(x-t)^{\tau}\right) d t\right] f(u) d u .
\end{aligned}
$$

Substituting $(x-t)=\lambda$ in the above equation, we get

$$
\begin{aligned}
& \left(R_{\alpha+; \tau, c}^{\omega ; a, b}\left[I_{\alpha+}^{\mu} f\right]\right)(x) \\
& \quad=\int_{u=\alpha}^{x} \frac{1}{\Gamma(\mu)}\left[\int_{\lambda=x-u}^{0}(\lambda)^{c-1}(x-\lambda-u)^{\mu-1}{ }_{2} R_{1}\left(a, b ; c ; \tau ; \omega(\lambda)^{\tau}\right)(-d \lambda)\right] f(u) d u \\
& =\int_{u=\alpha}^{x} \frac{1}{\Gamma(\mu)}\left[\int_{\lambda=0}^{x-u}(\lambda)^{c-1}(x-\lambda-u)^{\mu-1}{ }_{2} R_{1}\left(a, b ; c ; \tau ; \omega(\lambda)^{\tau}\right) d \lambda\right] f(u) d u .
\end{aligned}
$$

This is the proof of (31), and using the same procedure leads to the second identity of (30).

\section{Competing interests}

The authors declare that they have no competing interests.

\section{Authors' contributions}

All authors contributed equally to the manuscript. All authors read and approved the final manuscript.

\section{Author details}

'Department of Applied Mathematics, The M.S. University of Baroda, Vadodara, 390001, India. ${ }^{2}$ Department of Mathematical Sciences, Faculty of Applied Sciences, Charotar University of Science and Technology, Anand, Changa, 388421, India. ${ }^{3}$ Department of Mathematics, Shroff S.R. Rotary Institute of Chemical Technology, Dist. Bharuch, Vataria, 393002, India. ${ }^{4}$ Department of Applied Mathematics \& Humanities, S. V. National Institute of Technology, Surat, 395007, India.

\section{Acknowledgements}

We are very grateful to the anonymous referees for their careful reading and helpful comments.

\section{Received: 3 October 2013 Accepted: 26 March 2014 Published: 06 May 2014}

\section{References}

1. Kiryakova, V: Generalized Fractional Calculus and Applications. Wiley, New York (1994)

2. Mathai, AM, Saxena, RK: Generalized Hypergeometric Functions with Applications in Statistics and Physical Sciences. Springer, Berlin (1973)

3. Samko, SG, Kilbas, AA, Marichev, Ol: Fractional Integrals and Derivatives: Theory and Applications. Gordon \& Breach, Yverdon (1993)

4. Rainville, ED: Special Functions. Macmillan Co., New York (1960)

5. Erdélyi, A (ed.): Higher Transcendental Functions. McGraw-Hill, New York (1953)

6. Kilbas, AA, Saigo, M, Trujillo, Jj: On the generalized Wright function. Fract. Calc. Appl. Anal. 5, 437-460 (2004)

7. Virchenko, N, Kalla, SL, Al-Zamel, A: Some results on a generalized hypergeometric function. Integral Transforms Spec. Funct. 12(1), 89-100 (2001)

8. Virchenko, N, Lisetska, O, Kalla, SL: On some fractional integral operators involving generalized Gauss hypergeometric functions. Appl. Appl. Math. 5(10), 1418-1427 (2010) 
9. Virchenko, N, Rumiantseva, OV: On the generalized associated Legendre functions. Fract. Calc. Appl. Anal. 11(2), 175-185 (2008)

10. Rao, SB, Patel, AD, Prajapati, JC, Shukla, AK: Some properties of generalized hypergeometric function. Commun Korean Math. Soc. 28(2), 303-317 (2013)

11. Rao, SB, Shukla, AK: Note on generalized hypergeometric function. Integral Transforms Spec. Funct. 24(11), 896-904 (2013)

12. Prajapati, JC, Saxena, RK, Jana, RK, Shukla, AK: Some results on Mittag-Leffler function operator. J. Inequal. Appl. 2013, Article ID 33 (2013)

13. Rao, SB, Salehbhai, IA, Shukla, AK: On sequence of functions containing generalized hypergeometric function. Math. Sci. Res. J. 17(4), 98-110 (2013)

14. Rao, SB, Prajapati, JC, Shukla, AK: Wright type hypergeometric function and its properties. Adv. Pure Math. 3(3), 335-342 (2013)

15. Shukla, AK, Prajapati, JC: On a generalized Mittag-Leffler type function and generated integral operator. Math. Sci. Res. J. 12(12), 283-290 (2008)

16. Srivastava, HM, Tomovski, Z: Fractional calculus with an integral operator containing a generalized Mittag-Leffler functions in the kernel. Appl. Math. Comput. 211, 198-210 (2009)

17. Kilbas, AA, Srivastava, HM, Trujillo, JJ: A Theory and Applications of Fractional Differential Equations. North-Holland Mathematical Studies, vol. 204. Elsevier, Amsterdam (2006)

18. Hilfer, R (ed.): Application of Fractional Calculus in Physics. World Scientific, Singapore (2000)

19. Miller, KS, Ross, B: An Introduction to the Fractional Calculus and Fractional Differential Equations. Wiley, New York (1993)

20. Mathai, AM, Haubold, HJ: Special Functions for Applied Scientists. Springer, Berlin (2010)

21. Nyamoradi, N, Baleanu, D, Agarwal, RP: Existence and uniqueness of positive solutions to fractional boundary value problems with nonlinear boundary conditions. Adv. Differ. Equ.. 2013, Article ID 266 (2013)

22. Baleanu, D, Agarwal, RP, Mohammadi, H: Some existence results for a nonlinear fractional differential equation on partially ordered Banach spaces. Bound. Value Probl. 2013, Article ID 112 (2013)

23. Baleanu, D, Mustafa, OG, Agarwal, RP: Asymptotic integration of $(1+\alpha)$-order fractional differential equations. Comput. Math. Appl. 62(3), 1492-1500 (2011)

24. Srivastava, HM, Manocha, HL: A Treatise on Generating Functions. John Wiley/Ellis Horwood, New York/Chichester (1984)

25. Kilbas, AA: Fractional calculus of the generalized Wright function. Fract. Calc. Appl. Anal. 8(2), 113-126 (2005)

26. Gehlot, KS, Prajapati, JC: Fractional calculus of generalized k-Wright function. J. Fract. Calc. Appl. 4(2), $283-289$ (2013)

10.1186/1687-1847-2014-119

Cite this article as: Rao et al.: Some properties of Wright-type generalized hypergeometric function via fractional calculus. Advances in Difference Equations 2014, 2014:119

\section{Submit your manuscript to a SpringerOpen ${ }^{\circ}$ journal and benefit from:}

- Convenient online submission

Rigorous peer review

- Immediate publication on acceptance

- Open access: articles freely available online

- High visibility within the field

- Retaining the copyright to your article 